\title{
Apical Shear Stress Enhanced Organic Cation Transport in Human OCT2/MATE1-Transfected Madin-Darby Canine Kidney Cells Involves Ciliary Sensing
}

\author{
Aishwarya Jayagopal, Paul R. Brakeman, Peter Soler, Nicholas Ferrell, William Fissell, \\ Deanna L. Kroetz, and Shuvo Roy \\ Departments of Bioengineering and Therapeutic Sciences (A.J., P.S., D.L.K., S.R.) and Pediatrics (P.R.B.), University of California \\ San Francisco (UCSF), San Francisco, California; and Department of Medicine, Division of Nephrology, Vanderbilt University \\ Medical Center, Nashville, Tennessee (N.F., W.F.)
}

Received November 13, 2018; accepted February 27, 2019

\begin{abstract}
Active transport by renal proximal tubules plays a significant role in drug disposition. During drug development, estimates of renal excretion are essential to dose determination. Kidney bioreactors that reproduce physiologic cues in the kidney, such as flowinduced shear stress, may better predict in vivo drug behavior than do current in vitro models. In this study, we investigated the role of shear stress on active transport of 4-(4-(dimethylamino)styryl)-N-methylpyridinium iodide (ASP + ) by Madin-Darby canine kidney cells exogenously expressing the human organic cation transporters organic cation transporter 2 (OCT2) and multidrug and toxin extrusion protein 1 (MATE1). Cells cultured in a parallel plate under continuous media perfusion formed a tight monolayer with a high barrier to inulin. In response to increasing
\end{abstract}

levels of shear stress $\left(0.2-2\right.$ dynes $\left./ \mathrm{cm}^{2}\right)$, cells showed a corresponding increase in transport of $\mathrm{ASP}+$, reaching a maximal 4.2-fold increase at 2 dynes $/ \mathrm{cm}^{2}$ compared with cells cultured under static conditions. This transport was inhibited with imipramine, indicating active transport was present under shear stress conditions. Cells exposed to shear stress of $2 \mathrm{dynes} / \mathrm{cm}^{2}$ also showed an increase in RNA expression of both transfected human and endogenous OCT2 (3.7- and 2.0-fold, respectively). Removal of cilia by ammonium sulfate eliminated the effects of shear on ASP+ transport at 0.5 dynes $/ \mathrm{cm}^{2}$ with no effect on ASP+ transport under static conditions. These results indicate that shear stress affects active transport of organic cations in renal tubular epithelial cells in a cilia-dependent manner.

\section{Introduction}

Despite major advancements in preclinical methods for the selection of optimal drug candidates, drug development remains a long and expensive process that has a very low yield of marketed new molecular entities (Watkins, 2011). A major shortfall of preclinical models is the inability to predict clearance and toxicity in humans. Approximately $30 \%$ of drugs that are successful in preclinical studies fail in humans as the result of unanticipated clearance values or toxicities (Kola and Landis, 2004)

The kidney is a particularly important target for in vitro cell culture modeling because it is responsible for elimination of

This work was supported by National Institutes of Health National Institute of Biomedical Imaging and Bioengineering [Grants U01EB021214, R01EB014315] and the National Institute of Diabetes and Digestive and Kidney Diseases [Grant K01DK092357]; the Defense Threat Reduction Agency [Grant CBMXCEL-XL1-2-001]; the National Science Foundation Graduate Student Research Fellowship; and the Patterson-Barclay Foundation and the Wildwood Foundation.

This work was previously presented as an abstract at the American Society of Nephrology, Annual Meeting, Chicago, Il, 2016. Aishwarya Jayagopal, Shuvo Roy, Deanna L. Kroetz: Evaluation of Renal Cells for In Vitro Modeling of Proximal Tubule Drug Transport. J Am Soc Nephrol 27, 2016: 736A.

https://doi.org/10.1124/jpet.118.255026. more than a third of all drugs and most metabolites (Morrissey et al., 2013). The renal proximal tubule is important for drug testing during development because it performs most of the active transport of drug candidates and is particularly sensitive to toxic injury (Giacomini et al., 2010). The renal tubule is a monolayer of epithelial cells with filtrate flowing across the apical surface. A basement membrane underlies the tubular epithelium, and adjacent peritubular capillaries allow for reabsorption of water and solutes. The proximal tubule microenvironment, particularly fluid shear stress and apicobasal oncotic gradients, is incompletely reproduced by conventional cell culture techniques. Epithelial cell bioreactors that capture salient in vivo physiology may improve the accuracy of clearance and toxicity predictions derived from in vitro assays (Pfaller and Gstraunthaler, 1998; Astashkina et al., 2012).

Growing evidence indicates that proximal tubule cell morphology and functionality can vary with the growth environment, such as growth surface porosity, exposure to fluid on both sides, and/or fluid shear stress. Previous work from Essig and Friedlander (2003), as well as from our laboratory (Ferrell et al., 2010), has demonstrated that tubular fluid flow induced rearrangement in the apical actin cytoskeleton of proximal 
tubule cells (Ferrell et al., 2010). Subsequently, several studies have demonstrated that shear stress affects the expression levels and localization of multiple uptake and efflux transporters, including sodium-hydrogen antiporter 3 , $\mathrm{Na}^{+} / \mathrm{K}^{+}$-ATPase, glucose transporters, epithelial sodium channel, and the endocytosis receptors, megalin and cubulin (Duan et al., 2010; Raghavan et al., 2014; Jansen et al., 2016; Ernandez et al., 2018). Other work has demonstrated that shear flow in a tubular bioreactor system orients and elongates renal tubular cells along the flow path (Venzac et al., 2018). In vitro evidence indicates that some alterations in cytoskeletal structure and transport function are likely related to the mechanosensory function of cilia (Overgaard et al., 2009; Raghavan et al., 2014); however, few studies have considered the effect of shear stress on drug transporters in renal cells. Furthermore, little is known about the effect of different shear rates on cell functionality, particularly with sustained exposure to shear stress. Most studies have considered a single shear stress rate and have performed only short-term experiments (1-6 hours), making it difficult to distinguish between the true effects of shear and the cellular stress response to abrupt changes to the microenvironment (Duan et al., 2010; Jang et al., 2013; Maggiorani et al., 2015).

Here, a microfluidic bioreactor was used to examine the effect of graded levels of shear stress on the renal proximal tubule cell drug transporters organic cation transporter 2 (OCT2) and multidrug and toxin extrusion protein 1 (MATE1). We demonstrate that increasing apical shear stress leads to increasing organic cation transport and transporter expression and that cilia are involved in this cellular response to shear stress.

\section{Materials and Methods}

Device Fabrication and Assembly. We have previously published our design for a parallel plate bioreactor that provides a fluid flow path of adjustable height across the apical side of the cells and a static reservoir on the basal side (Brakeman et al., 2016). For the work presented here, we adapted our previous design to create a multiplexed device with four separate flow paths to allow for testing four biologic conditions at once (Fig. 1). Each device is composed of three layers: a base with a 5-ml apical reservoir, a middle plate to hold the Snapwell insert (Costar, Corning, Corning, NY) with $1.12-\mathrm{cm}^{2}$ cell area and a top plate containing a 1- to 2-ml basolateral reservoir. The plates were machined from polysulfone to allow for sterilization by autoclaving. Laser-cut silicone gaskets of 500- to $1000-\mu \mathrm{m}$ height were sandwiched between each set of plates to seal the fluid compartments and on the apical side to define the channel height. The bioreactor was assembled using screws. The media reservoir and bubble trap were a built-in column. The inlets and outlets were connected using barbed connectors to Masterflex LS-14 silicone tubing with 1.6-mm inner diameter (Cole Parmer Lab Supplies, Vernon Hills, IL). Fluid flow and thus apical shear stress were set and controlled by a peristaltic pump (Cole Parmer).

Cell Culture and Flow. MDCK cells transfected with either an empty vector or a pair of uptake and efflux transporters (hOCT2/ hMATE1) (König et al., 2011) were provided by Dr. Martin Fromm (Erlangen, Germany) and cultured in minimum essential medium with Earle's balanced salt solution (UCSF Cell Culture Facility) with $10 \%$ fetal bovine serum (Gibco, Thermo Fisher Scientific, Waltham, MA) and $1 \%$ penicillin-streptomycin (UCSF Cell Culture Facility). Five hundred micrograms per milliliter hygromycin (UCSF Cell Culture Facility) and $100 \mathrm{mg} / \mathrm{ml}$ geneticin (Sigma-Aldrich, St. Louis, MO) were added to the media for the double transfected cells. For flow experiments, hOCT2/hMATE1 MDCK cells were plated on the underside of Snapwell inserts (Corning) at a density of 300,000 cells/well $\left(250,000\right.$ cells $\left./ \mathrm{cm}^{2}\right)$, grown under static conditions until confluence, and then placed in the bioreactor. Apical media flow was increased over 7 days from 0.1 to 1-6 $\mathrm{ml} / \mathrm{min}$ until desired shear stress was achieved and then left stable for 72 hours before experimentation. Cells were grown under static conditions for the same amount of time as controls.

Immunofluorescence. Cells were fixed with $4 \%$ paraformaldehyde (Thermo Fisher Scientific), permeabilized with $0.1 \%$ Triton-X in PBS (Sigma-Aldrich), and blocked with bovine serum albumin (SigmaAldrich). They were subsequently incubated with 1:50 dilution of Alexa 488-labeled zonula occludens-1 mouse monoclonal antibody (Life Technologies, Carlsbad, CA) or a primary acetylated $\alpha$-tubulin mouse monoclonal antibody (Life Technologies) for 60 minutes. Cells treated with the $\alpha$-tubulin antibody were then incubated with a secondary 561 anti-mouse goat antibody (Life Technologies) and phalloidin (Thermo Fisher Scientific) for F-actin staining. Imaging was performed using a Nikon (Tokyo, Japan) spectral confocal microscope with a $40 \times$ oil objective.

Barrier Performance. Inulin, a 5000 Da polymer, is a wellknown marker of glomerular filtration rate and is a marker of the leakiness of the proximal tubule in vivo (Sohtell et al., 1983):

$$
\text { Barrier performance }=\frac{\mathrm{C}_{\text {apical }}-\mathrm{C}_{\text {basal }}}{\mathrm{C}_{\text {apical }}}
$$

FITC-labeled inulin (Sigma-Aldrich) was added to the apical media and allowed to flow through the devices. Samples were collected from the basal reservoir every 24 hours and analyzed for inulin content
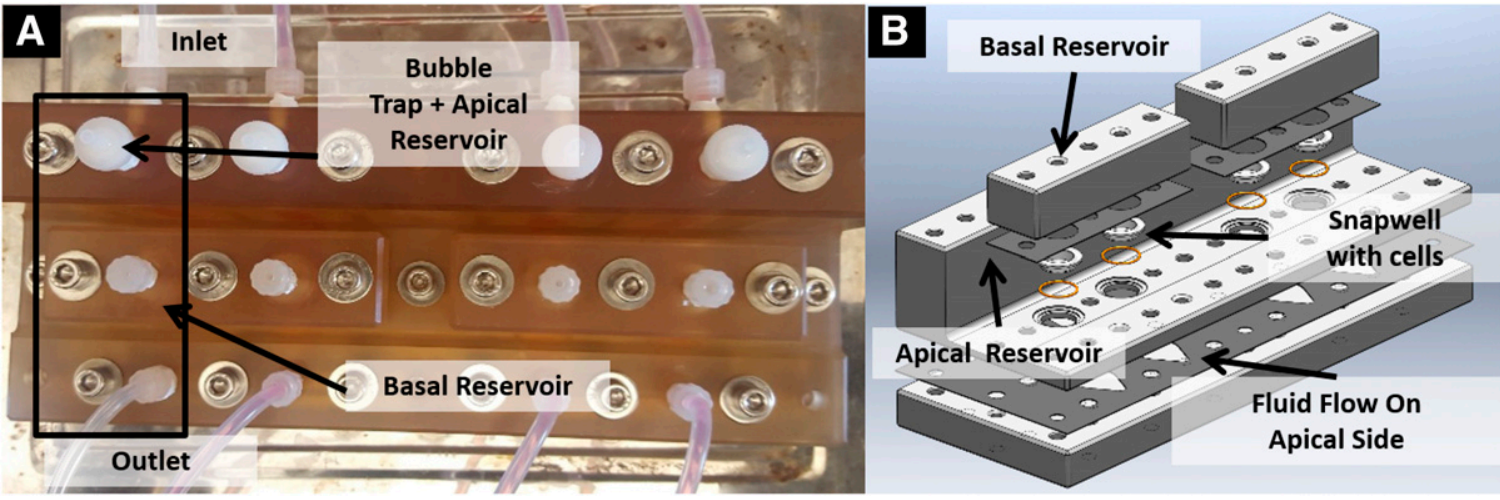

Fig. 1. Bioreactor design. (A) Top-down view of the four-channel device. Boxed area indicates one channel with inlet, 5-ml apical reservoir with bubble trap, outlet, and a 1- to 2-ml basal reservoir. (B) Exploded schematic of the four-channel device set up with 1.12- $\mathrm{cm}^{2} \mathrm{cell}$ area, gasket (500-1000 $\mu$ m), defined apical fluid flow path, and basal reservoir are shown. 
using a Genios Pro fluorescence plate reader (Tecan, Mannedorf, Switzerland). Barrier function of the cell monolayer was calculated from inulin levels measured in the apical and basal compartments as described by eq. 1 . Here, $C_{\text {apical }}$ is the concentration of inulin in the apical compartment, and $C_{\text {basal }}$ is the concentration in the basal compartment. Inulin leak was calculated as concentration in the basal (donor) compartment divided by the cell area after 24 hours.

ASP+ Transport. Transduced transporter genes were induced with $10 \mathrm{mM}$ Na-butyrate (Sigma-Aldrich) 24 hours before a transport experiment, as described previously (König et al., 2011). When appropriate, cells were incubated with an inhibitor (500 $\mu \mathrm{M}$ imipramine or $1 \mathrm{mM}$ cimetidine (Sigma-Aldrich)) on the basal side for 30 minutes, followed by the addition of $25 \mu \mathrm{M}$ 4-(4-dimethylamino)styryl-N-methylpyridinium (ASP+) (Life Technologies) and incubation for 1 hour. Samples were collected from the apical and basal media. Cells were then rinsed with ice-cold phosphate-buffered saline three times and lysed for measurement of ASP + accumulation and transport. Transport experiments were simultaneously performed on cells under shear stress and cultured under static conditions. ASP + concentration was quantified on a Genios Pro fluorescence plate reader (Tecan) at an excitation wavelength of $485 \mathrm{~nm}$ and an emission wavelength of $590 \mathrm{~nm}$.

Protein Quantification. Cells were lysed with 1\% SDS-10 M $\mathrm{NaOH}$ lysis buffer while shaking overnight. Protein content was measured using a standard Pierce BCA protein assay kit (Thermo Fisher). Where appropriate, protein content was normalized to cell growth area.

RNA Expression. RNA was extracted from cells using an RNeasy RNA Extraction Kit (Qiagen, Hilden, Germany), and cDNA was generated using an iScript kit (Bio-Rad, Hercules, CA). cDNA was used for detection of human and dog OCT2 and MATE1 and dog P-glycoprotein (P-gp) by quantitative reverse transcriptasepolymerase chain reaction using a Taqman assay and probes (Applied Biosystems) on the Fast Realtime PCR instrument (Applied Biosystems). Ribosomal protein S18 (RS-18) was used as housekeeping control. The effect of shear stress on transporter expression was analyzed using the $\Delta \Delta \mathrm{Ct}$ method using transporter levels expressed relative to RS-18 (Peters et al., 2007; Schmittgen and Livak, 2008). P-gp was used as a measure of global effects of shear stress on transporter expression.

Deciliation. Cells were grown to confluence and then incubated in the absence or presence of $30 \mathrm{mM}$ ammonium sulfate (Fluka AG) for 24 hours before measurement of ASP + transport (Overgaard et al., 2009). The presence of cilia was determined by imaging of $\alpha$-tubulin as described already herein.

Statistics. All experiments were performed in triplicate with a minimum of two technical replicates within each experiment. Data are expressed as mean \pm S.D. and graphed as box and whisker plots. Statistical analyses were performed by unpaired one-way or two-way analysis of variance, and a $P$ value of $<0.05$ was considered significant. Data were analyzed using Prism Version 6.0 (Graphpad, San Diego, CA).

\section{Results}

Cell Morphology and Barrier Function. Immunofluorescence analysis of cells placed under flow revealed uniform monolayer formation with the tight-junction protein, zonula occludens-1, localized to the tight junctions between cells under both static and flow conditions (Fig. 2, A-C). Quantification of total protein content demonstrated up to a 1.6-fold increase in protein content per square centimeter of monolayer in response to shear stress (Fig. 2D). Next, we measured the permeability of inulin, a marker of proximal tubule leakiness, across the monolayer. Devices retained a barrier performance rate of $97.9 \% \pm 1.42 \%$ over 7 days of culture under up to 2 dynes $/ \mathrm{cm}^{2}$ of shear stress (Fig. $2 \mathrm{E}$ ), resulting in a final inulin leak rate on day 6 of $0.13-0.69 \mu \mathrm{g} / \mathrm{cm}^{2}$ per day.

Effect of Shear Stress on Organic Cation Transport. Transport of ASP+, an auto-fluorescent substrate of OCT2 and MATE1, was measured for 1 hour at differing levels of shear stress $\left(0.2-2\right.$ dynes $\left./ \mathrm{cm}^{2}\right)$. MDCK cells exogenously expressing hOCT2 and hMATE1 showed a 4.2-fold increase in ASP+ transport in response to shear stress of 2 dynes $/ \mathrm{cm}^{2}$ as reflected in measures of both cellular accumulation (Fig. 3A) and transcellular transport (Fig. 3B). The effect of shear stress on ASP+ accumulation or transcellular transport was similar under shear stress of 0.5 and 2 dynes $/ \mathrm{cm}^{2}$. To determine whether active ASP + transport was increased, transport of ASP + by cells exposed to 0.2 dynes $/ \mathrm{cm}^{2}$ of shear stress was measured with or without pretreatment with $500 \mu \mathrm{M}$ imipramine, an OCT2- and MATE1-specific inhibitor. ASP+ transport was inhibited by imipramine $60.3 \% \pm 15.8 \%$ under shear stress conditions compared with $47.6 \% \pm 19.7 \%$ under static conditions (Fig. 3C).

To further understand this observation, the effect of shear stress on the expression of human OCT2 (transfected), canine OCT2 (endogenous), and canine P-gp (endogenous) was measured in cells exposed to varying levels of shear for 72 hours after a slow ramping-up of shear over 7 days (Fig. 4). In comparison with cells cultured under static conditions, MDCK cells exposed to shear showed increased expression of transfected human and endogenous OCT2 (up to 3.7- and 2.0fold, respectively), with no significant effect on the expression of transfected MATE1 or endogenous P-gp.

Role of Cilia in Response to Shear. Imaging showed that double-transfected MDCK cells express cilia under both static and flow conditions (Fig. 5, A and E). Exposure of the cells to $30 \mathrm{mM}$ ammonium sulfate for 24 hours caused a complete loss of cilia (Fig. 5, C and G). Importantly, deciliation had no gross effects on cell-membrane junctions, as measured by imaging of $\mathrm{F}$-actin, which anchors tight-junction proteins at the cell-membrane junctions in epithelial cells (Fig. 5, D and H) (Stevenson and Begg, 1994). Although there is some thickening of the F-actin staining in these images, this was not a consistent finding, and thus we did not quantitate this effect. Deciliation had no effect on transport by cells cultured under static conditions, but it completely eliminated the effects of shear stress on ASP+ transport in cells exposed to 0.5 dynes $/ \mathrm{cm}^{2}$ of shear (Fig. 6 ).

\section{Discussion}

The kidney plays a central role in the elimination of drugs in the human body. It is important to mimic accurately the complexity of renal physiology for in vitro drug testing, particularly with respect to fluid flow. Shear stress from fluid flow has been shown to affect cell morphology and ion transporters in vitro, but little is known about the effects on drug transporters (Duan et al., 2010; Ferrell et al., 2012; Jansen et al., 2016). Additionally, most previous studies have measured only the effect of short-term shear (1-6 hours), which is unlikely to fully isolate shear stress effects from other cellular stress responses (Duan et al., 2010; Jang et al., 2013; Maggiorani et al., 2015). In these studies, we focused on the effects of sustained (7 days) shear stress from fluid flow on drug transport to better mimic the sustained shear stress experienced by renal tubular cells in vivo. 

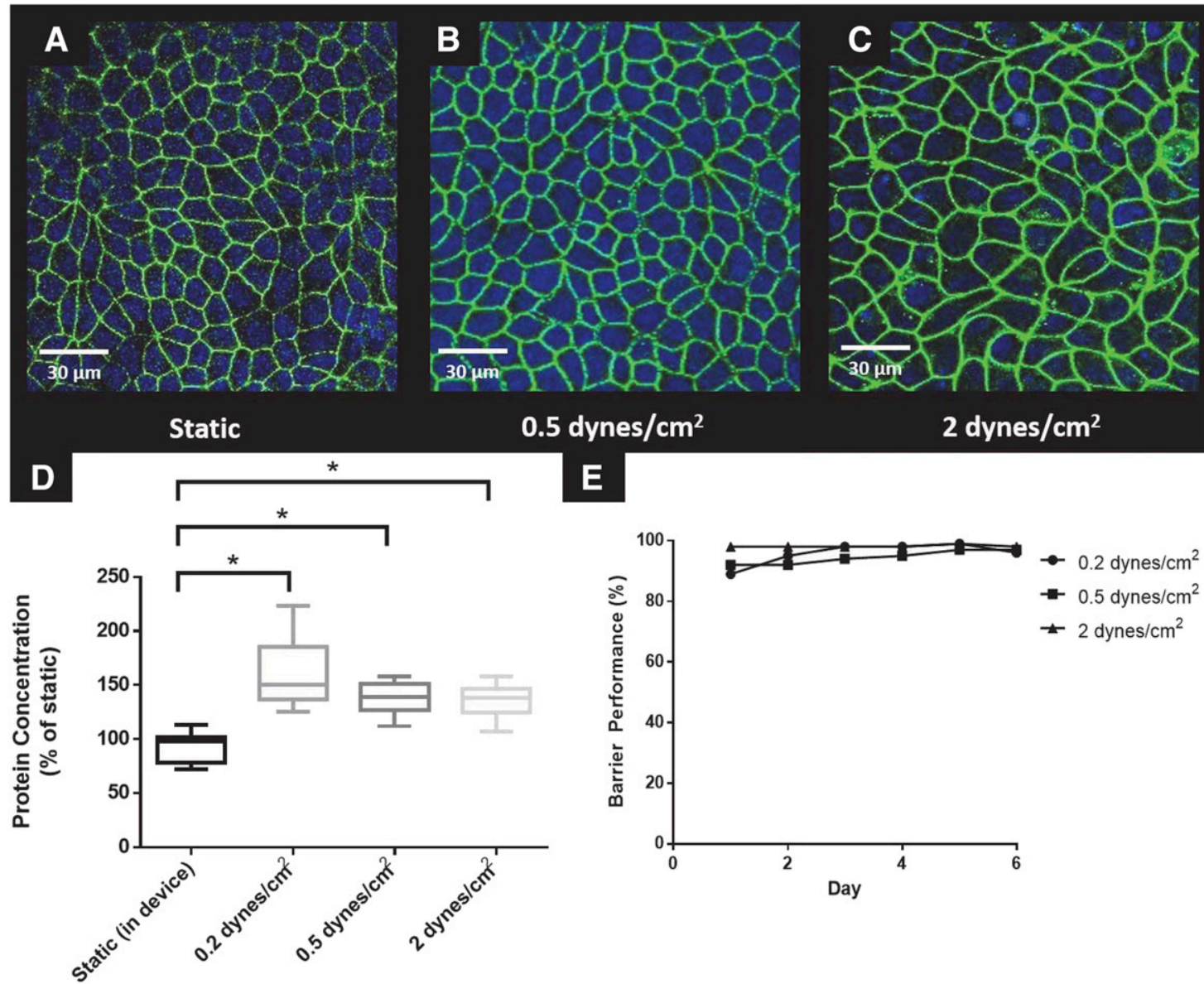

E

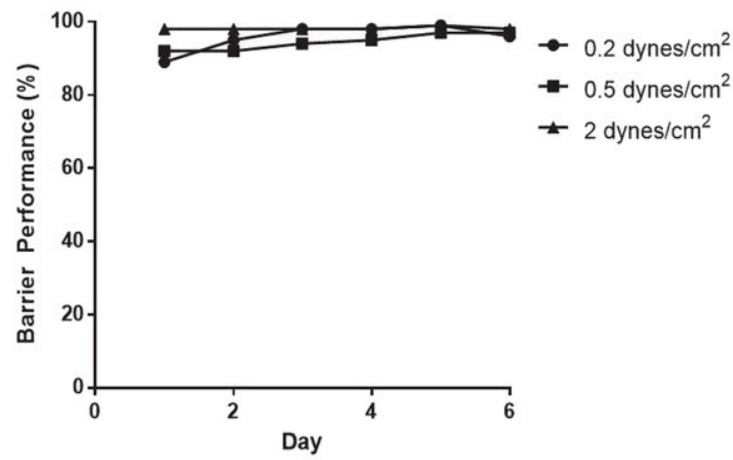

Fig. 2. Effects of shear stress on MDCK cell monolayer formation. (A-C) Nucleus (4',6-diamidino-2-phenylindole (DAPI)) and tight-junction Zonulaoccludens-1 (ZO-1) stains of cells under (A) no, (B) 0.5-, and (C) 2.0-dynes $/ \mathrm{cm}^{2}$ levels of shear stress. Shear stress was slowly ramped up to target shear stress over 7 days and then held stable for 72 hours before experimentation. Blue $=$ DAPI for nucleus and green $=$ ZO-1. (D) Protein content of cells under varying flow conditions. Shear stress was slowly ramped up to target shear stress over 7 days and then held stable for 72 hours before experimentation. Experiments were performed in triplicate, and data are shown as box (median with 25th to 75th percentile) and whiskers (minimum and maximum); ${ }^{*} P<0.05$. (E) Barrier performance of cells under flow as measured by inulin permeability across the monolayer every 24 hours for 6 days. Images presented are from a single representative device at each shear stress level.

Understanding how drug transporters respond to sustained shear stress can improve in vitro predictions of in vivo drug handling by the kidney. Accurately designed in vitro models of the kidney may standardize preclinical testing and reduce drug failure rates.

In this work, a parallel plate microfluidic bioreactor was used to determine the effects of sustained shear stress on organic cation transport by OCT2 and MATE1 in a renal tubule cell line. Several cell lines were considered for these studies, including human cell lines such as human proximal tubule cells from the Hopfer group (Orosz et al., 2004) and RPTECs (CRL-4031; American Type Culture Collection, Manassas, VA) (Wieser et al., 2008), but the doubletransfected hOCT2/hMATE1 MDCK cells were considered the most appropriate choice for this study for several reasons. First, the MDCK cells consistently demonstrate robust attachment to the transwell inserts, which allows them to withstand the initial stress of fluid flow. Second, these cells form tight monolayers, which prevents leakiness and is essential for the study of transcellular transport of marker substrates. Finally, exogenous expression of transporters allows for improved detection of active transport and the effect of perturbations.
Cells placed under flow formed a confluent monolayer and localized a tight-junction protein to the periphery of the cell. They also retained high barrier function as measured by inulin permeability. The ability of the epithelial cells to form a monolayer that prevents fluid and protein leak is extremely important for proper function of the tubule. Here, the leak through the MDCK cells was minimal at less than $1 \mu \mathrm{g} / \mathrm{cm}^{2}$ per day and significantly lower than that through human cells. We have previously shown that human kidney cell monolayers have a $10-20 \mu \mathrm{g} / \mathrm{cm}^{2}$ per day inulin leak (Brakeman et al., 2016), supporting the conclusion that the hOCT2/hMATE1 MDCK cells formed a robust monolayer.

Transport of ASP + was significantly increased in hOCT2/ hMATE1 MDCK cells exposed to varying levels of shear stress for 72 hours compared with static controls. ASP + is taken up by OCT2 (SLC22A2) and effluxed by MATE1 (SLC47A1), two organic cation transporters that work in concert to facilitate the renal secretion of commonly used drugs, such as metformin and cisplatin (Biermann et al., 2006; Wittwer et al., 2013). Similar effects of shear stress have been reported for proximal tubule ion transporters. Increases in albumin uptake, ion reabsorption, and megalin and cubulin expression and function have been reported in response to increased shear stress 

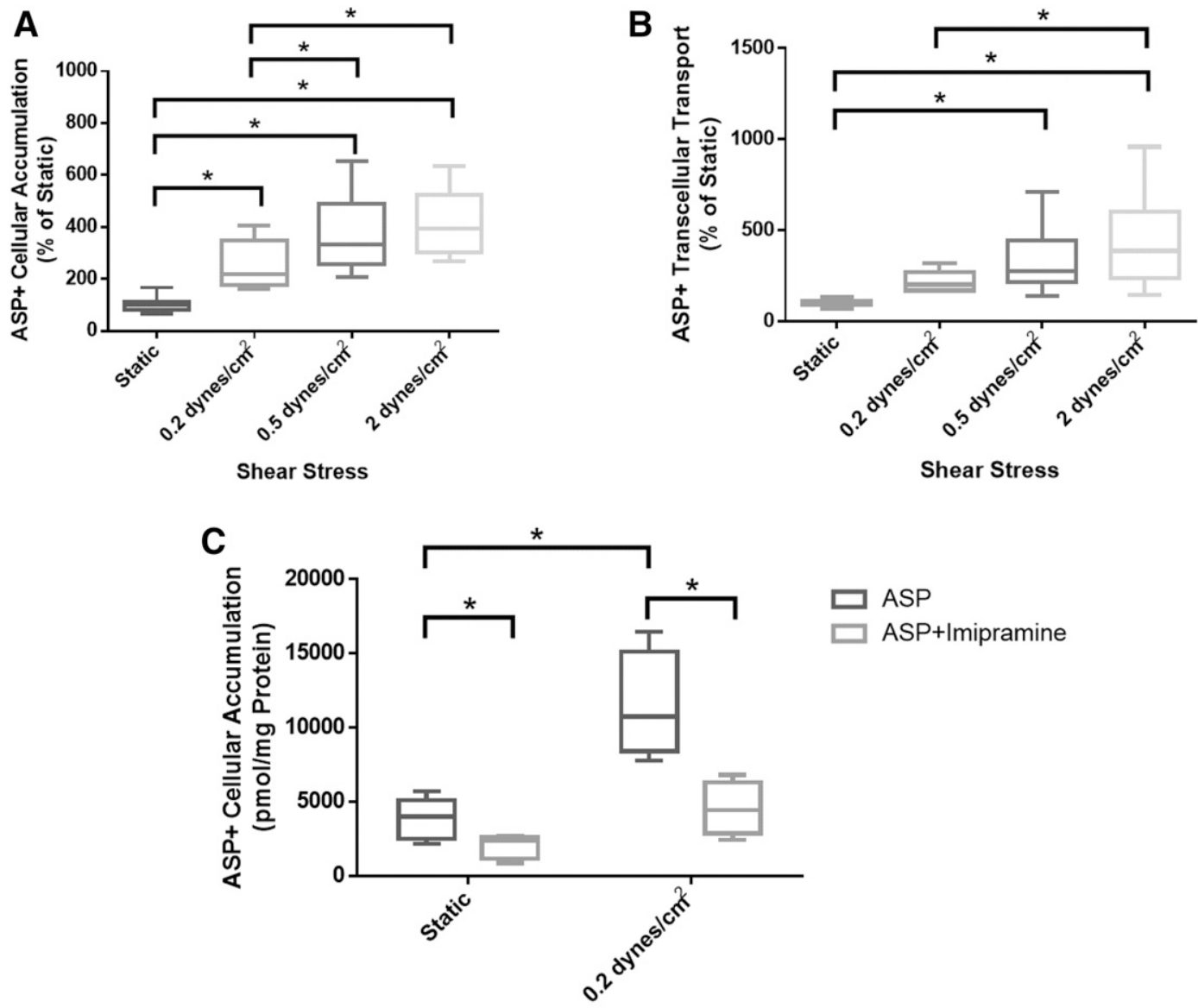

Shear Stress

Fig. 3. ASP+ transport in hOCT2/hMATE1 double-transfected MDCK cells exposed to shear stress. (A) Accumulation and (B) transcellular transport of ASP+ by a monolayer of cells exposed to $0-2$ dynes $/ \mathrm{cm}^{2}$ of shear stress for 72 hours, followed by basolateral addition of ASP+ for 1 hour. Shear stress was slowly ramped up to target shear stress over 7 days and then held stable for 72 hours before experimentation. Data are expressed in percent relative to accumulation or transport by cells cultured under static conditions. (C) ASP+ accumulation into cells exposed to $0-0.2 \mathrm{dynes} / \mathrm{cm}^{2}$ of shear stress for 72 hours, followed by basolateral addition of ASP+ for 1 hour, with or without an exposure to imipramine, an OCT/MATE1 inhibitor. Experiments were performed in triplicate and data are shown as box (median with 25 th to 75 th percentile) and whiskers (minimum and maximum); ${ }^{*} P<0.05$.

(Overgaard et al., 2009; Duan et al., 2010; Ferrell et al., 2012; Raghavan et al., 2014). Exposure of renal proximal tubule cells to shear stress is also associated with reduced apoptosis and faster recovery from acute cisplatin toxicity and enhanced inhibition of organic anion transport (Jang et al., 2013). Since shear stress from fluid flow is constantly present in the proximal tubule, these findings collectively support the use of more physiologic in vitro model systems to predict renal drug disposition and toxicity. It is noteworthy that there is no significant difference in transporter function between 0.5 and 2.0 dynes $/ \mathrm{cm}^{2}$ of shear stress. Prior studies by Essig and Friedlander (2003) had found that a minimum level of shear of $0.17 \mathrm{dynes} / \mathrm{cm}^{2}$ is required to elicit an alteration in cell morphology, and other studies have measured effects on physiology at up to $1 \mathrm{dyne} / \mathrm{cm}^{2}$ (Duan et al., 2010). This is the first study to explore the effects of a range of higher and sustained shear stress levels on functionality of renal drug transporters, and it is possible that the magnitude of biologic difference between the 0.5 and 2.0 dynes $/ \mathrm{cm}^{2}$ of shear stress that we evaluated was not large enough to allow for identification of differences between these levels of shear stress in our assays. Although further investigation is required, our results shed light on the required conditions for physiologically relevant models and the effects of increased shear stress levels owing to disease on renal drug handling.

One of the limitations of our experimental methods is that we only addressed transport at $\mathrm{pH} 7.4$ using media with $\mathrm{pH}$ 7.4 on both apical and basal sides mimicking the experimental methods previously described for the hOCT2/hMATE1 MDCK cells (Konig et al., 2011). An apical to basal pH gradient can affect OCT2 and MATE1 function, and there is typically a $\mathrm{pH}$ gradient under physiologic conditions (Muller et al., 2013). Our methods therefore limited our ability to evaluate the interaction between an apical to basal $\mathrm{pH}$ gradient and shear stress on OCT2 an MATE1 function. Since we used consistent $\mathrm{pH}$ of 7.4 throughout all experimental conditions, this lack of a $\mathrm{pH}$ gradient is unlikely to have affected our findings but potentially limits the applicability of our findings to OCT2 and MATE1 transport in the presence of an apical to basal $\mathrm{pH}$ gradient.

Surprisingly, the mRNA expression of transfected human and endogenous OCT2 was increased in cells exposed to all levels of shear stress compared with static controls. This upregulation of transporter expression was specific, with no measurable effect on endogenous P-gp expression or transfected MATE1. The upregulation of transporter expression 


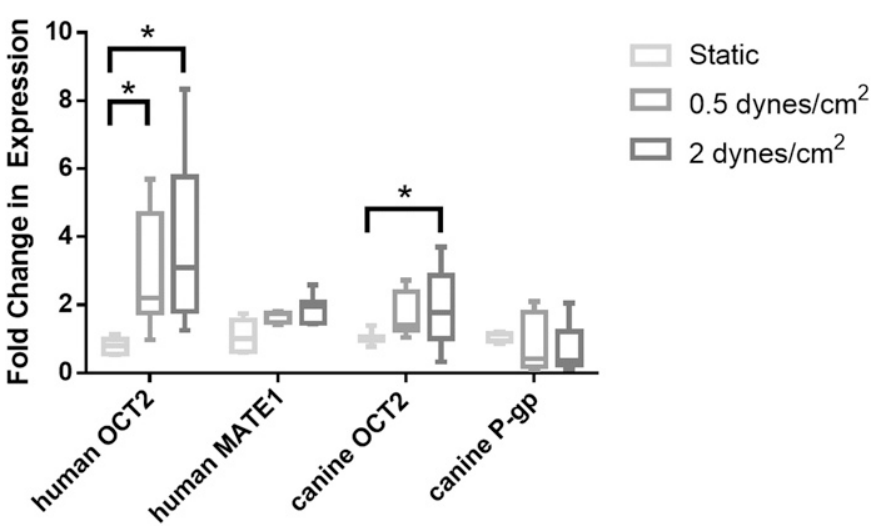

Fig. 4. Effect of shear stress on RNA expression of human (transfected) and endogenous canine OCT2 (organic cation transporter), transfected human MATE1, and canine P-gp in hOCT2/hMATE1 double-transfected MDCK cells. Cells were exposed to 0-2 dynes $/ \mathrm{cm}^{2}$ for 72 hours after slowly ramping up to target shear stress over 7 days before mRNA expression was measured. Experiments were performed in triplicate, and data are shown as box (median with 25th to 75 th percentile) and whiskers (minimum and maximum); $* P<0.05$

gives insight into the mechanism behind the increased transport and may be a result of enhanced mRNA stability or increased mRNA transcription. It is interesting to note that both the transfected and endogenous transporters were upregulated, which is unexpected since transfected OCT2 was expressed under a cytomegalovirus promoter and should not be subject to endogenous gene expression regulatory mechanisms. Although surprising, this observation is not unprecedented. A similar effect on OAT1 transfected proximal tubule cells exposed to perfusion has been reported, but the mechanism for the increased expression remains unclear (Jansen et al., 2016). In another study, Nrf2 signaling was found to play a role in increasing endogenous MATE2-K expression in response to shear stress (Fukuda et al., 2017). Further study into this is warranted and will be the subject of future investigations.

We also identified that the introduction of apical shear flow increased protein content per square centimeter of cells. This effect was the same magnitude for both 0.5 and $2 \mathrm{dynes} / \mathrm{cm}^{2}$ of shear, similar to the effect seen for mRNA expression and $\mathrm{ASP}+$ transport. There is some evidence that shear stress induces taller renal epithelial cells, which would result in a larger volume of cells per square centimeter and thus a likely increase in protein per square centimeter; however, this previously published phenomenon was only a minor observation in a larger body of data and not quantitated (Long et al., 2017). The increase in total protein in cells grown in the presence of apical shear flow may represent the normal protein content for healthy renal tubular epithelial cells that can be achieved only by the exposure of the cells to apical shear flow. Therefore, the lower levels of protein content in cells grown in static culture may represent an abnormal cellular state as could occur clinically in situations of low apical filtrate flow such as acute kidney injury. This phenomenon deserves further study.

Cilia are known to have mechanosensory roles in the proximal tubule (Raghavan and Weisz, 2016). Therefore, to determine whether they play a critical role in the increased OCT2 and MATE1-mediated transport reported here, the effect of cilia removal on transporter function was measured. The complete blockade of shear-dependent increases in ASP+ transport by removal of cilia in our studies is similar to the effect found in studies by Raghavan et al. (2014), who demonstrated a cilia-dependent upregulation in endocytosis

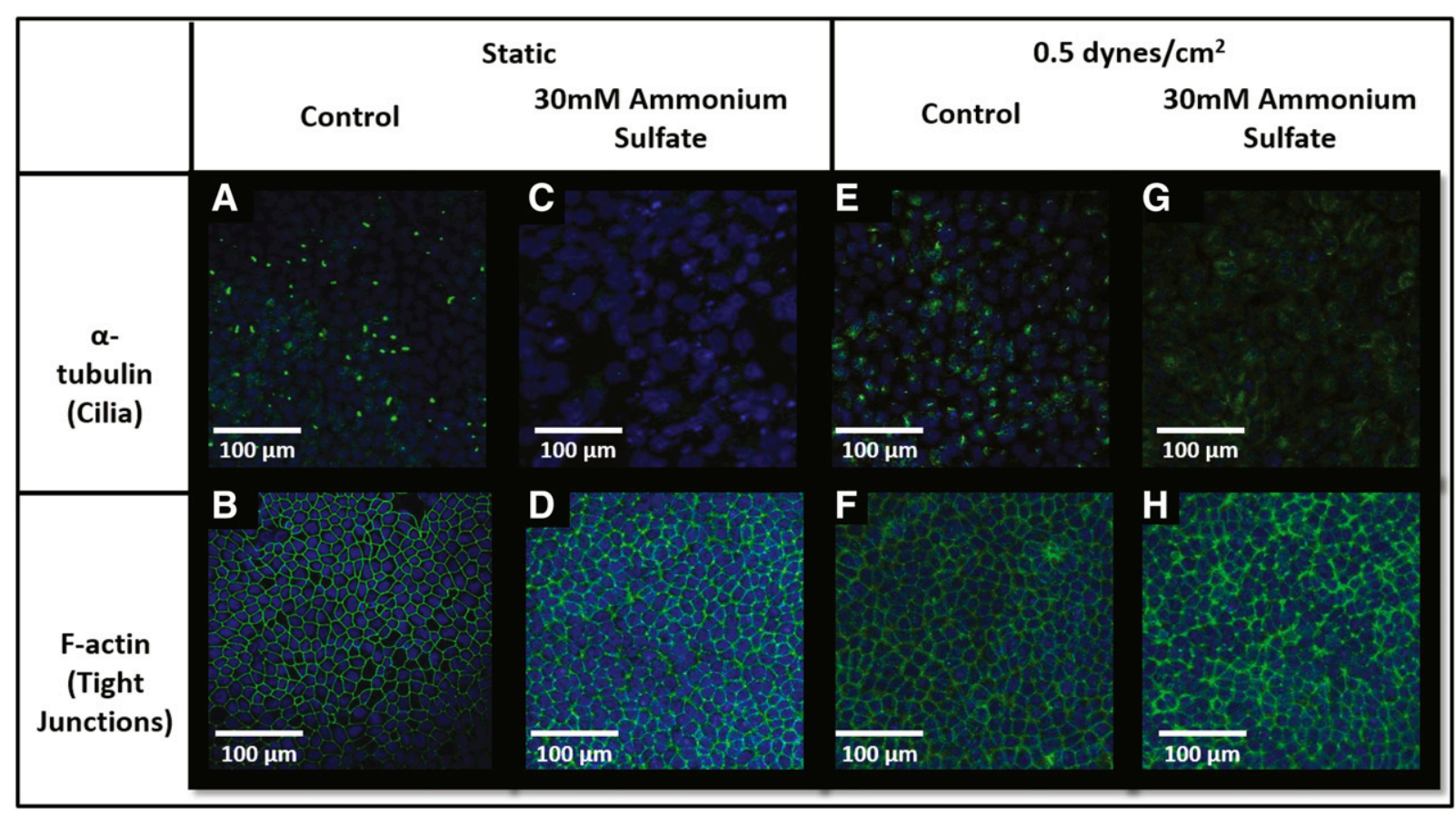

Fig. 5. Effect of deciliation on morphology of hOCT2/hMATE1 MDCK cells. Staining of nuclei (DAPI) and cilia ( $\alpha$-tubulin) or tight junctions (F-actin) in cells exposed to $0-0.5$ dynes $/ \mathrm{cm}^{2}$ for 72 hours after slowly ramping up to target shear stress over 7 days and, where labeled, $30 \mathrm{mM}$ ammonium sulfate for 24 hours. (A, C, E, and G). Blue $=$ DAPI, green $=\alpha$-tubulin; $(\mathrm{B}, \mathrm{D}, \mathrm{F}$, and H) blue $=$ DAPI, green $=$ F-actin 
A

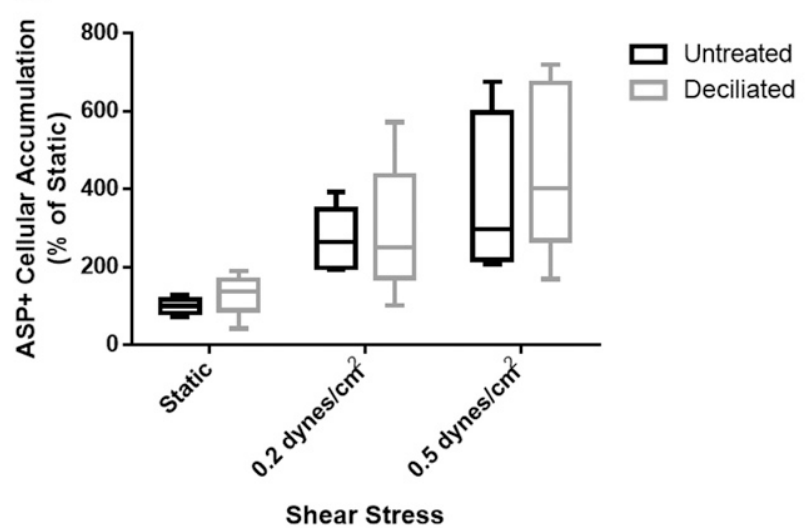

B

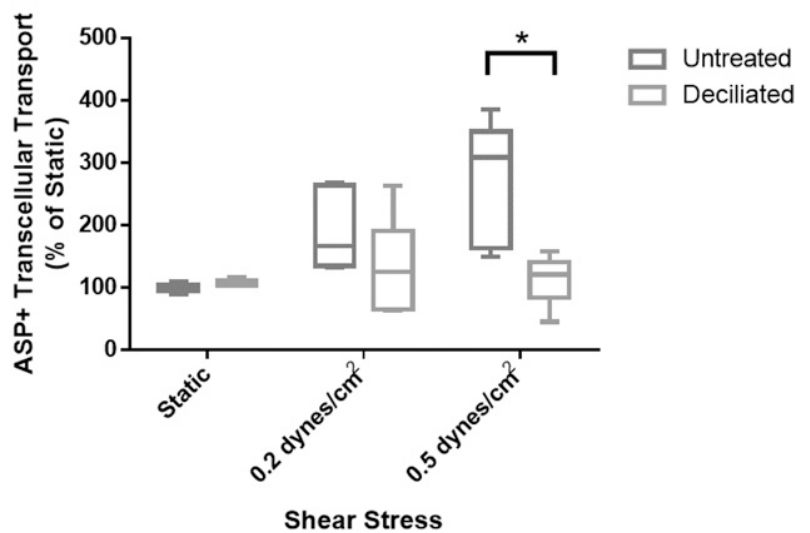

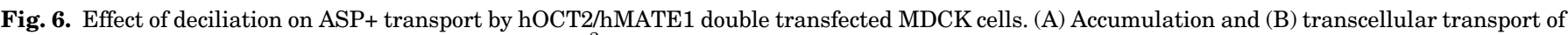

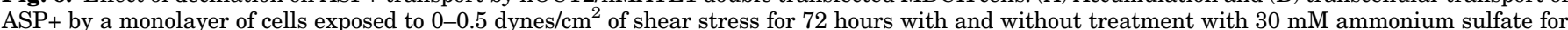

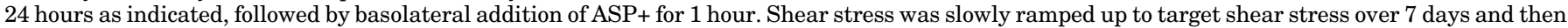

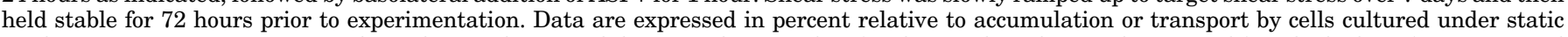

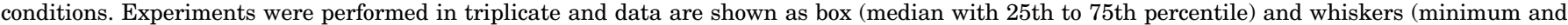
maximum); $* P<0.05$.

in response to fluid flow. Ammonium sulfate likely has other uncharacterized effects on cell behavior and functionality that may indirectly impact solute transport. Despite possible offtarget effects, this method of deciliation does test the role of ciliary sensing on transporter-medicated movement of organic solutes. The mechanism of signaling between the mechanosensory proteins in the cilia and the transporter is currently unknown. One hypothesis is that the function of the organic cation transporters is changed by altered ion transport. Removal of cilia is known to alter solute motility in the proximal tubule cells, specifically by reducing $\mathrm{Na}^{+} / \mathrm{K}^{+}$ATPase membrane localization and modifying paracellular transport (Overgaard et al., 2009). It is possible that this results in alterations in MATE1 function, an antiporter dependent on the $\mathrm{H}^{+}$gradient. Another hypothesis is that sensing of shear stress affects expression of organic cation transporters. Shear stress modulates MATE2-K function through Nrf2 signaling (Fukuda et al., 2017). Other organic cation transporters may respond similarly to shear stress, which would be eliminated when the mechanosensory cilia are removed. Much of this is unknown and warrants further study. Interestingly, cilia removal did not have a significant effect on ASP + transport by cells exposed to $0.2 \mathrm{dynes} / \mathrm{cm}^{2}$ of shear stress, but a robust effect was observed when shear stress was increased to $0.5 \mathrm{dynes} / \mathrm{cm}^{2}$. This suggests that the cilia sense a threshold shear stress level; in turn, cilia signal changes in transporter expression and function before measurable effects on transport are expected. Overall, the dependence of organic cation transport on ciliary sensing of shear stress provides insight into the mechanosensory signaling pathway involved and the minimum level of stress that might be required to trigger a robust response to shear.

In summary, these data demonstrate that shear stress from fluid flow has a significant effect on organic cation transporter function and expression in MDCK cells. Furthermore, upregulation of organic cation transport was dependent on the presence of cilia. We propose that apical shear stress is an important component of any in vitro modeling of renal tubular cells and is likely to be an important component of modeling the renal secretory clearance and nephrotoxicity of drugs. The specific mechanism by which mechanical stress signals increased transporter activity and expression is still unclear and requires further study.

\section{Acknowledgments}

We thank Martin Fromm for the gift of hOCT2/hMATE1 transfected cells and Alex Ideguchi, Niecholle Rocco, and Sneha Krishna for assistance with the project.

\section{Authorship Contributions}

Participated in research design: Jayagopal, Brakeman, Soler, Ferrell, Fissell, Kroetz, Roy.

Conducted experiments: Jayagopal, Brakeman.

Contributed new reagents or analytic tools: Konig, Fromm.

Performed data analysis: Jayagopal.

Wrote or contributed to the writing of the manuscript: Jayagopal, Brakeman, Soler, Ferrell, Fissell, Kroetz, Roy.

\section{References}

Astashkina A, Mann B, and Grainger DW (2012) A critical evaluation of in vitro cell culture models for high-throughput drug screening and toxicity. Pharmacol Ther 134:82-106.

Biermann J, Lang D, Gorboulev V, Koepsell H, Sindic A, Schröter R, Zvirbliene A Pavenstädt H, Schlatter E, and Ciarimboli G (2006) Characterization of regulatory mechanisms and states of human organic cation transporter 2. Am J Physiol Cell Physiol 290:C1521-C1531.

Brakeman P, Miao S, Cheng J, Lee C-Z, Roy S, Fissell WH, and Ferrell N (2016) A modular microfluidic bioreactor with improved throughput for evaluation of polarized renal epithelial cells. Biomicrofluidics 10:064106.

Duan Y, Weinstein AM, Weinbaum S, and Wang T (2010) Shear stress-induced changes of membrane transporter localization and expression in mouse proximal tubule cells. Proc Natl Acad Sci USA 107:21860-21865.

Ernandez T, Udwan K, Chassot A, Martin P-Y, and Feraille E (2018) Uninephrectomy and apical fluid shear stress decrease ENaC abundance in collecting duct principal cells. Am J Physiol Renal Physiol 314:F763-F772.

Essig M and Friedlander G (2003) Tubular shear stress and phenotype of renal proximal tubular cells. J Am Soc Nephrol 14 (Suppl 1):S33-S35.

Ferrell N, Desai RR, Fleischman AJ, Roy S, Humes HD, and Fissell WH (2010) A microfluidic bioreactor with integrated transepithelial electrical resistance (TEER) measurement electrodes for evaluation of renal epithelial cells. Biotechnol Bioeng 107:707-716.

Ferrell N, Ricci KB, Groszek J, Marmerstein JT, and Fissell WH (2012) Albumin handling by renal tubular epithelial cells in a microfluidic bioreactor. Biotechnol Bioeng 109:797-803.

Ferrell N, Ricci KB, Desai RR, Groszek J, Marmerstein JT, and Fissell WH (2012) A microfluidic bioreactor for epithelial cell studies under fluid shear stress. FASEB $J$ 26:911.3.

Fukuda Y, Kaishima M, Ohnishi T, Tohyama K, Chisaki I, Nakayama Y, OgasawaraShimizu M, and Kawamata Y (2017) Fluid shear stress stimulates MATE2-K expression via Nrf2 pathway activation. Biochem Biophys Res Commun 484:358-364. Giacomini KM, Huang S-M, Tweedie DJ, Benet LZ, Brouwer KLR, Chu X, Dahlin A Evers R, Fischer V, Hillgren KM, et al.; International Transporter Consortium 
(2010) Membrane transporters in drug development. Nat Rev Drug Discov 9: 215-236.

Jang K-J, Mehr AP, Hamilton GA, McPartlin LA, Chung S, Suh K-Y, and Ingber DE (2013) Human kidney proximal tubule-on-a-chip for drug transport and nephrotoxicity assessment. Integr Biol 5:1119-1129.

Jansen J, Fedecostante M, Wilmer MJ, Peters JG, Kreuser UM, van den Broek PH, Mensink RA, Boltje TJ, Stamatialis D, Wetzels JF, et al. (2016) Bioengineered kidney tubules efficiently excrete uremic toxins. Sci Rep 6:26715.

Kola I and Landis J (2004) Can the pharmaceutical industry reduce attrition rates? Nat Rev Drug Discov 3:711-715.

König J, Zolk O, Singer K, Hoffmann C, and Fromm MF (2011) Double-transfected MDCK cells expressing human OCT1/MATE1 or OCT2/MATE1: determinants of uptake and transcellular translocation of organic cations. Br J Pharmacol 163:546-555.

Long KR, Shipman KE, Rbaibi Y, Menshikova EV, Ritov VB, Eshbach ML, Jiang Y, Jackson EK, Baty CJ, and Weisz OA (2017) Proximal tubule apical endocytosis is modulated by fluid shear stress via an mTOR-dependent pathway. Mol Biol Cell 28:2508-2517.

Maggiorani D, Dissard R, Belloy M, Saulnier-Blache J-S, Casemayou A, Ducasse L, Grès S, Bellière J, Caubet C, Bascands J-L, et al. (2015) Shear stress-induced alteration of epithelial organization in human renal tubular cells. PLoS One 10:e0131416.

Morrissey KM, Stocker SL, Wittwer MB, Xu L, and Giacomini KM (2013) Renal transporters in drug development. Annu Rev Pharmacol Toxicol 53:503-529.

Müller F, König J, Hoier E, Mandery K, and Fromm MF (2013) Role of organic cation transporter OCT2 and multidrug and toxin extrusion proteins MATE1 and MATE2-K for transport and drug interactions of the antiviral lamivudine. Biochem Pharmacol 86:808-815.

Orosz DE, Woost PG, Kolb RJ, Finesilver MB, Jin W, Frisa PS, Choo C-K, Yau C-F, Chan $\mathrm{K}-\mathrm{W}$, Resnick MI, et al. (2004) Growth, immortalization, and differentiation potential of normal adult human proximal tubule cells. In Vitro Cell Dev Biol Anim 40:22-34.

Overgaard CE, Sanzone KM, Spiczka KS, Sheff DR, Sandra A, and Yeaman C (2009) Deciliation is associated with dramatic remodeling of epithelial cell junctions and surface domains. Mol Biol Cell 20:102-113.

Peters IR, Peeters D, Helps CR, and Day MJ (2007) Development and application of multiple internal reference (housekeeper) gene assays for accurate normalisation of canine gene expression studies. Vet Immunol Immunopathol 117:55-66.
Pfaller W and Gstraunthaler G (1998) Nephrotoxicity testing in vitro--what we know and what we need to know. Environ Health Perspect 106 (Suppl 2): $559-569$

Raghavan V, Rbaibi Y, Pastor-Soler NM, Carattino MD, and Weisz OA (2014) Shear stress-dependent regulation of apical endocytosis in renal proximal tubule cells mediated by primary cilia. Proc Natl Acad Sci USA 111:8506-8511.

Raghavan V and Weisz OA (2016) Discerning the role of mechanosensors in regulating proximal tubule function. Am J Physiol Renal Physiol 310:F1-F5.

Schmittgen TD and Livak KJ (2008) Analyzing real-time PCR data by the comparative C(T) method. Nat Protoc 3:1101-1108.

Sohtell M, Karlmark B, and Ulfendahl H (1983) FITC-inulin as a kidney tubule marker in the rat. Acta Physiol Scand 119:313-316.

Stevenson BR and Begg DA (1994) Concentration-dependent effects of cytochalasin D on tight junctions and actin filaments in MDCK epithelial cells. J Cell Sci 107: $367-375$

Venzac B, Madoun R, Benarab T, Monnier S, Cayrac F, Myram S, Leconte L, Amblard F, Viovy J-L, Descroix S, et al. (2018) Engineering small tubes with changes in diameter for the study of kidney cell organization. Biomicrofluidics 12: 024114 .

Watkins PB (2011) Drug safety sciences and the bottleneck in drug development. Clin Pharmacol Ther 89:788-790.

Wieser M, Stadler G, Jennings P, Streubel B, Pfaller W, Ambros P, Riedl C, Katinger H, Grillari J, and Grillari-Voglauer R (2008) hTERT alone immortalizes epithelial cells of renal proximal tubules without changing their functional characteristics. Am J Physiol Renal Physiol 295:F1365-F1375.

Wittwer MB, Zur AA, Khuri N, Kido Y, Kosaka A, Zhang X, Morrissey KM, Sali A, Huang Y, and Giacomini KM (2013) Discovery of potent, selective multidrug and toxin extrusion transporter 1 (MATE1, SLC47A1) inhibitors through prescription drug profiling and computational modeling. J Med Chem 56:781-795.

Address correspondence to: Paul R. Brakeman, Department of Pediatrics, University of California, San Francisco, 550 16th Street, Floor 5, San Francisco, CA 94143-3214. E-mail: Paul.brakeman@ucsf.edu 